
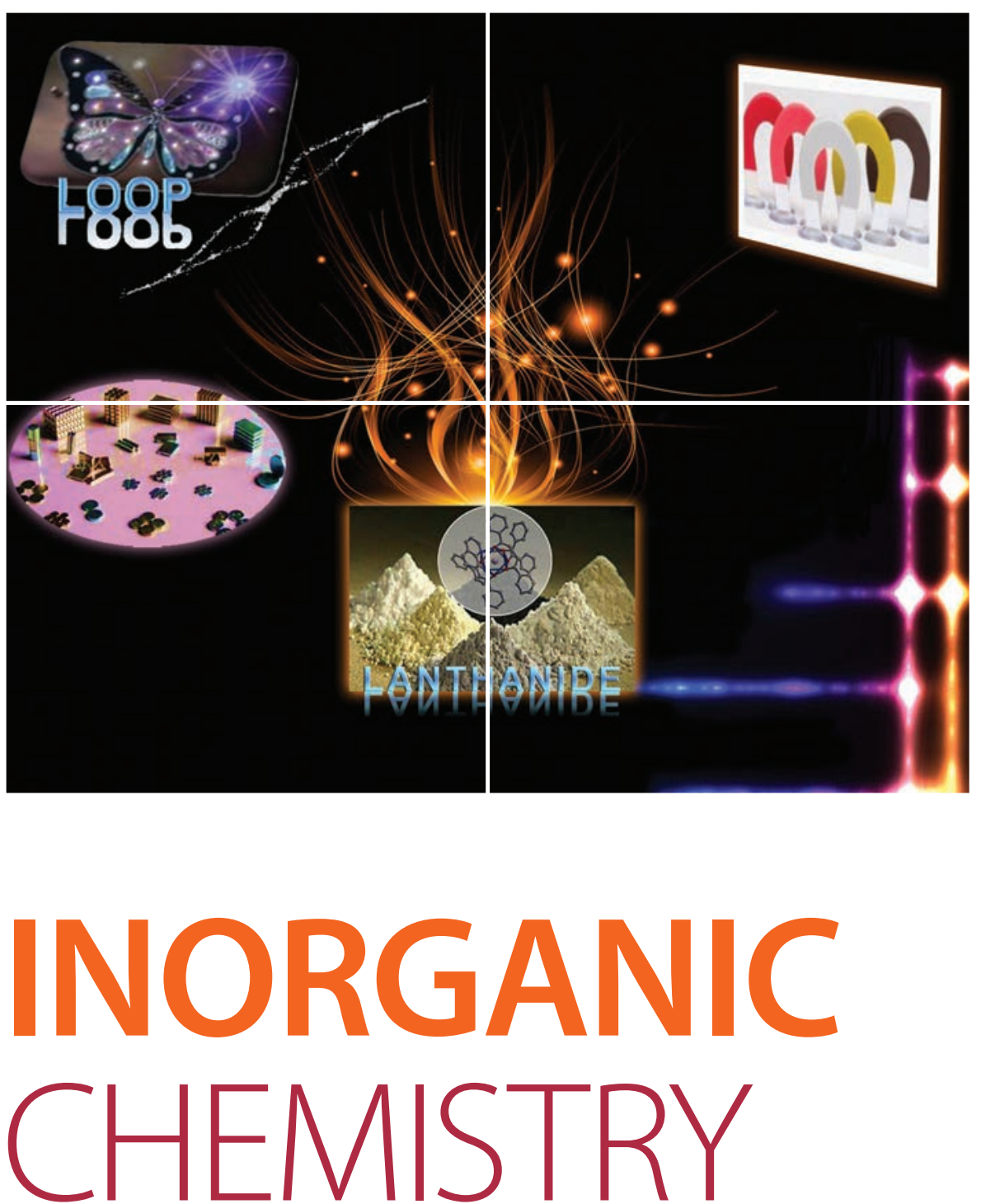

\title{
FRONTIERS
}




\title{
RESEARCH ARTICLE
}

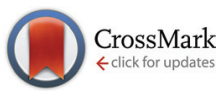

Cite this: Inorg. Chem. Front., 2015, 2, 854

Received 31st May 2015,

Accepted 7th July 2015

DOI: 10.1039/c5qi00090d

rsc.li/frontiers-inorganic

\section{Chiral biomolecule based dodecanuclear dysprosium(III)-copper(II) clusters: structural analyses and magnetic properties $\uparrow$}

\author{
Biplab Joarder, ${ }^{a}$ Soumya Mukherjee, ${ }^{a}$ Mahendra Patil, ${ }^{a}$ Shufang Xue, ${ }^{\mathrm{b}}$ Jinkui Tang ${ }^{\star b}$ \\ and Sujit K. Ghosh*a
}

\begin{abstract}
An isostructural family of three dodecanuclear chiral $\mathrm{M}_{4} \mathrm{Cu}_{8}(\mathrm{M}=\mathrm{Dy}, \mathrm{Y})$ complexes has been synthesized by adopting a mixed-ligand strategy, wherein one of the constituent linkers, pyroglutamic acid, a recognized biomolecule, has been credibly functioning as the chiral precursor imparting the chirality to the aforementioned complex. The highly symmetric new-fangled $\mathrm{M}_{4} \mathrm{Cu}_{8}$ coordination-core, comprising of four square-symmetrically coordinated Dy/Y-vertex sharing $\mathrm{M}_{2} \mathrm{Cu}_{2}$ cubane units, has been synthesized as an unprecedented discrete coordination complex, which has been analysed by magnetic measurements.
\end{abstract}

\section{Introduction}

Discrete coordination architectures of diverse nuclearities have steadily drawn the attention of chemists and materials scientists for the intriguing functional aspects emanating from the simple design principles based on coordination chemistry driven self-assembly processes involving metal nodes and a vast spectrum of numerous linkers. ${ }^{1-6}$ Among these, coordination architectures with excellent symmetric features have been the most investigated ones owing to their captivating coordination environments, giving rise to utilitarian attributes as a consequence of their symmetry-aided structure-property correlation. $^{7-13}$ The inherent symmetry in such complexes makes the structure-property correlation much easier to predict, leading to a strategic rationale based design principle for attaining the target properties and an insight thereinto. Over the decades, employment of a binary or ternary combination of linkers for yielding such complexes has proved quite an efficient protocol. Since the multiple coordination sites involved with their distinct denticities and coordination geometries, in unison with the associated non-covalent inter-

${ }^{a}$ Indian Institute of Science Education and Research (IISER), Pune. Dr Homi Bhabha Road, Pashan, Pune, India 411008. E-mail: sghosh@iiserpune.ac.in;

Fax: +91 202589 8022; Tel: +912025908076

${ }^{b}$ State Key Laboratory of Rare Earth Resource Utilization, Changchun Institute of Applied Chemistry, Chinese Academy of Sciences, Changchun, 130022, China.

E-mail:tang@ciac.ac.cn

$\dagger$ Electronic supplementary information (ESI) available: Ligand structures for $\mathrm{L}$ and D enantiomers, IR and EDX data (for metal analyses) for all three reported compounds, along with crystal data and structure refinement for compound $\mathbf{1 L}$. CCDC 1403754 for 1L. For ESI and crystallographic data in CIF or other electronic format see DOI: 10.1039/c5qi00090d actions, govern the crucial nuclearity aspect for such species; more than one appropriately functionalized linker is frequently employed for obtaining coordination complexes promising from the standpoint of application. ${ }^{14-25}$ Higher nuclearity-lanthanide discrete complexes or clusters have been the most sought-after ones considering the flurry of such materials evolved in the last decade exhibiting slow magnetic relaxation-triggered single molecule magnet (SMM) characteristics, ${ }^{26-31}$ ubiquitously imperative behind the development of novel molecular nanomagnets functioning as miniaturized devices for high-density information storage, molecule spintronics and quantum computing. ${ }^{32-40}$

Biomolecules can be expediently employed as the building blocks of functional coordination complexes considering their biocompatibility, structural diversity, intrinsic self-assembly characteristics via their different metal-binding sites, low cost and ample availability coupled with individual chiral signatures of the precursor biomolecule-based synthons. ${ }^{41-45}$ The chiral features with multidentate facets particularly make such biomolecule-based coordination complexes an exciting domain to investigate, because of their prospective biological applications, including drug delivery or intracellular imaging. Although much effort has been devoted to lanthanide (4f)-based and lanthanide-transition (3d-4f)-based coordination complexes, resulting into the discovery of noteworthy magnetic properties such as slow magnetic relaxation-triggered single molecule magnet behaviour, magnetic refrigeration etc.; biomolecule based coordination complexes ${ }^{46}$ have never been the focus from the standpoint of magnetism phenomena, in spite of the recently explored spectrum of applications presented by this emerging class of materials. ${ }^{46-52}$ Despite some recent reports, pyroglutamic acid (PGA) or pidolic acid, a scar- 
cely found proline derivative recently commercialized as a dietary supplement, wherein the free amino group of glutamic acid or glutamine undergoes cyclization to form a lactam, is yet to be comprehensively harnessed for coordination complex reactions. ${ }^{53-57}$ Importantly, this oxo-derivative of a secondary amino acid and a noteworthy metabolite in the glutathione cycle is a vital biomolecule possessing memory-enhancement effect, hair-follicle growth and dermal penetration enhancing consequences. ${ }^{58-60}$ Hence, the employment of PGA in the construction of $3 \mathrm{~d}-4 \mathrm{f}$ based coordination complexes seems quite a daunting task, considering the unification of the dual features of biocompatibility and magnetism in a single discrete coordination entity. This indeed seems crucial from the standpoint of exploring magnetic materials with the requisite biocompatibility. ${ }^{61,62}$

Furthermore, to synthesize such desired coordination complexes based on hard Lewis acid (PGA) with high hydrooxophilic $\mathrm{Ln}^{\mathrm{III}}$ and $\mathrm{Cu}^{\mathrm{II}}$ ions, ${ }^{63-69}$ we have purposefully chosen pyridine-based auxiliary ligands with flexible alkoxide ends, which might act as chelating and bridging ligands, owing to hard Lewis base $\mathrm{O}$ and $\mathrm{N}$ binding sites. Herein, we have exploited the oxophilic biomolecule-linker PGA (L and D enantiomers) with ancillary ligand 2-(2-hydroxyethyl)pyridine (HEP) (Fig. $\mathrm{S} 1 \dagger$ ) for the synthesis of a series of three isostructural, chiral biomolecule based $\mathrm{M}_{4} \mathrm{Cu}_{8}$ coordination complexes $\left[\mathrm{M}_{4} \mathrm{Cu}_{8}(\mathrm{HEP})_{8}(\mathrm{PGA})_{8}(\mathrm{OH})_{8}\left(\mathrm{NO}_{3}\right)_{4}\right](\mathrm{M}=$ Dy and $\mathrm{Y})$ and have analyzed their magnetism properties with the aid of direct and alternate current susceptibility measurements by SQUID (superconducting quantum interference device). The difference brought about by the presence of the high-anisotropy Dy (III) centres, while coupled with $\mathrm{Cu}(\mathrm{II})$ centres in this aforementioned core, was to be compared with the isostructural $\mathrm{Y}_{4} \mathrm{Cu}_{8}$ core, highlighting the role of $3 \mathrm{~d}-4 \mathrm{f}$ interactions in the Dy-analogue in exhibiting magnetic properties.

\section{Experimental section}

\section{Materials and measurements}

All the reagents and solvents were commercially available and were used without further purification. The powder X-ray patterns (PXRD) were recorded on Bruker D8 Advanced X-ray diffractometer at room temperature using $\mathrm{Cu} K \alpha$ radiation $(\lambda=$ $1.5406 \AA$ A). FT-IR spectra were measured on NICOLET 6700 FT-IR Spectrophotometer using $\mathrm{KBr}$ pellets.

Synthesis of $\left[\mathrm{Dy}_{4} \mathrm{Cu}_{8}(\mathrm{HEP})_{8}(\mathrm{~L}-\mathrm{PGA})_{8}(\mathrm{OH})_{8}\left(\mathrm{NO}_{3}\right)_{4}\right](1 \mathrm{~L})$. To a sonicated methanolic solution $(5 \mathrm{~mL})$ of $\mathrm{HEP}(11.3 \mu \mathrm{L}$, $0.1 \mathrm{mmol}$ ) and $\mathrm{NaOH}$ ( $4 \mathrm{mg}, 0.1 \mathrm{mmol}$ ), another well-sonicated solution of PGA (16.1 mg, $0.125 \mathrm{mmol}$ ) and $\mathrm{NaOH}$ ( $5 \mathrm{mg}, 0.125 \mathrm{mmol}$ ) dissolved in $5 \mathrm{~mL} \mathrm{MeOH}$ was added. A $15 \mathrm{~mL}$ binary solvent mixture $(1: 2)$ of $\mathrm{MeOH}$ and $\mathrm{MeCN}$ was further added to the aforementioned reaction mixture, following which solid $\mathrm{Cu}\left(\mathrm{NO}_{3}\right)_{2} \cdot 3 \mathrm{H}_{2} \mathrm{O}(12.1 \mathrm{mg}, 0.05 \mathrm{mmol})$ and $3 \mathrm{ml}$ of $0.03 \mathrm{mmol}(13.6 \mathrm{mg}$ ) methanolic solution of Dy $\left(\mathrm{NO}_{3}\right)_{3} \cdot x \mathrm{H}_{2} \mathrm{O}$ is slowly added while sonicating continuously. Slow evaporation of solvent mixture over the next ten days with no mechanical disturbance, yielded excellent quality blue cube-shaped single crystals of compound $\left[\mathrm{Dy}_{4} \mathrm{Cu}_{8}(\mathrm{HEP})_{8}{ }^{-}\right.$ $\left.(\mathrm{L}-\mathrm{PGA})_{8}(\mathrm{OH})_{8}\left(\mathrm{NO}_{3}\right)_{4}\right](\mathbf{1 L})$, suitable for single crystal X-ray analysis. Yield $\sim 60 \%$. IR (KBr, $\left.\mathrm{cm}^{-1}\right): 3644(\mathrm{~s}), 2845(\mathrm{~m}), 2719(\mathrm{~m})$, 2407(m), 2086(w), 1709(b), 1428(w), 1291(w), 1234(w), 1160(m), 1088(w), 1033(s), 971(w), 879(s), 779(s), 652(s) (Fig. S2†). Anal. Calcd (found) for $\mathrm{C}_{96} \mathrm{H}_{112} \mathrm{Cu}_{8} \mathrm{Dy}_{4} \mathrm{~N}_{16} \mathrm{O}_{40}$ : C, 35.06 (35.38); H, 3.43 (2.98); N, 6.82 (6.65)\%.

Synthesis of $\left[\mathrm{Dy}_{4} \mathrm{Cu}_{8}(\mathrm{HEP})_{8}(\mathrm{D}-\mathrm{PGA})_{8}(\mathrm{OH})_{8}\left(\mathrm{NO}_{3}\right)_{4}\right]$ (1D) and $\left[\mathbf{Y}_{4} \mathrm{Cu}_{8}(\mathrm{HEP})_{8}(\mathrm{~L}-\mathrm{PGA})_{8}(\mathbf{O H})_{8}\left(\mathrm{NO}_{3}\right)_{4}\right] \quad(2 \mathrm{~L})$. A similar reaction protocol as mentioned for $1 \mathbf{L}$ was followed for the syntheses of $1 D$ from D-PGA (instead of L-PGA) and Dy $\left(\mathrm{NO}_{3}\right)_{3} \cdot x \mathrm{H}_{2} \mathrm{O} ; 2 \mathrm{~L}$ from L-PGA and $\mathrm{Y}\left(\mathrm{NO}_{3}\right)_{3} \cdot 6 \mathrm{H}_{2} \mathrm{O}$ (replacing $\mathrm{Dy}\left(\mathrm{NO}_{3}\right)_{3} \cdot x \mathrm{H}_{2} \mathrm{O}$ ). The similar PXRD nature for the bulk phases of these two compounds along with the similar IR stretching frequencies and elemental analyses reinstates the isostructural nature for these two compounds, with respect to compound 1L. Repeated trials to obtain the D-PGA based analogous compound to $2 \mathbf{L}$ could not be obtained due to encountered precipitation issues, hence has not been included in the report.

IR ( $\left.\mathrm{KBr}, \mathrm{cm}^{-1}\right)$ for 1D: 3634(s), 2663(w), 2720(w), 2401(m), 2083(w), 1701(b), 1426(b), 1292(m), 1159(sh), 1088(w), 1034(s), 968(w) (Fig. S2 $\dagger$ ). IR (KBr, $\mathrm{cm}^{-1}$ ) for 2L: 3630(s), 2848(m), 2729(m), 2391(m), 2080(w), 1699(b), 1443(w), 1295(w), 1234(w), 1155(m), 1083(w), 1037(s), 967(w), 880(s), 778(s), 653(s) (Fig. S2†). Anal. Found for 1D (\%): C, 35.13; H, 3.11; N, 6.90. Anal. Found for $2 \mathrm{~L}(\%)$ : C, 35.29; H, 3.57; N, 6.77.

\section{X-ray structural studies}

Single-crystal X-ray data of compound 1L was collected at $200 \mathrm{~K}$ on a Bruker KAPPA APEX II CCD Duo diffractometer (operated at $1500 \mathrm{~W}$ power: $50 \mathrm{kV}, 30 \mathrm{~mA}$ ) using graphitemonochromated Mo $\mathrm{K} \alpha$ radiation $(\lambda=0.71073 \AA$ ). A cubeshaped blue crystal was mounted using nylon CryoLoops (Hampton Research) with Paratone-N (Hampton Research). The data integration and reduction were processed with SAINT $^{70}$ software. A multi-scan absorption correction was applied to the collected reflections. The structure was solved by the direct method using SHELXTL ${ }^{71}$ and was refined on $F^{2}$ by full-matrix least-squares technique using the SHELXL- $97^{72}$ program package within the WINGX ${ }^{73}$ programme. All nonhydrogen atoms were refined anisotropically. All hydrogen atoms were located in successive difference Fourier maps and they were treated as riding atoms using SHELXL default parameters. The structures were scrutinized by the Adsym subroutine of PLATON $^{74}$ to ensure that no additional symmetry could be applied to the models. Crystal data and structure refinement details for complex $\mathbf{1 L}$ is summarized in Table S1. $\dagger$

\section{Magnetic measurement details}

Magnetic susceptibility measurements were carried out on a Quantum Design MPMS-XL7 SQUID magnetometer equipped with a $7 \mathrm{~T}$ magnet. The direct current $(\mathrm{dc})$ measurements were collected with an external magnetic field of 1000 Oe in the temperature range $1.9-300 \mathrm{~K}$, and the alternating-current (ac) 
measurements were carried out in a 3.0 Oe ac field oscillating at $1000 \mathrm{~Hz}$ in the temperature range $2-40 \mathrm{~K}$. The experimental magnetic susceptibility data are corrected for the diamagnetism estimated from Pascal's tables and sample holder calibration. ${ }^{75}$

\section{Results and discussion}

Compounds 1L and $\mathbf{2 L}$ derived from L-PGA, and 1D derived from D-PGA were prepared at room temperature by slow evaporation of the respective reaction mixtures, as described in the Experimental section. Single crystal X-ray analysis reveals that compound 1L crystallized in the tetragonal space group P4222 with $Z=1$. The molecular structure of compound $\mathbf{1} \mathbf{L}$, showing the central square-shaped $\mathrm{Dy}_{4}$ core flanked by four $\mathrm{Dy}_{2} \mathrm{Cu}_{2}$ cubanes at each of the central Dy-centres is shown in Fig. 1; the precise arrangement of which is shown in sharper detail in Fig. 2a. The $3 \mathrm{~d}-4 \mathrm{f}$ heterometallic dodecahedron $\left(\mathrm{Dy}_{4} \mathrm{Cu}_{8}\right)$ is highly symmetric, since all the cubanes and each of the analogous elements therein are found to have crystallographically identical features (Fig. 2a). The four cubanes are interconnected via the Dy(III) vertices in a typical vertex-sharing arrangement. Interestingly, considering the entire bimetallic central core, only one type of Dy(III): Dy1, one kind of $\mathrm{Cu}(\mathrm{II})$ : $\mathrm{Cu} 1$, and just two different types of $\mathrm{O}$ atoms (O1 and O5) constitute the entire assemblage of $\mathrm{Dy}_{4} \mathrm{Cu}_{8} \mathrm{O}_{16}$, providing testimony to the symmetric attributes of this cluster core. While O1 centers are found to connect the two different metals Dy(III) and $\mathrm{Cu}(\mathrm{II}), \mathrm{O} 5$ centers act as bridging oxo-linkers between the $4 \mathrm{f}-\mathrm{Dy}(\mathrm{III})$ apices of this bimetallic core.

The bond angles and intermetallic distances spanned across each of the solitary cubane units have been represented in Fig. 2b. Considering the homometallic pairs; while the two larger Dy(III) centers are separated by a distance of $3.87 \AA$, at the very central core of the motif, the distant $\mathrm{Cu}$ (II) centers are in quite close proximity (3.22 $\AA$ ), resulting in the observed sets of acute and obtuse angles across the metal-vertices, characteristic of a typical cubane. Since the $\mathrm{Cu}_{2} \mathrm{O}_{2}$ and $\mathrm{Dy}_{2} \mathrm{O}_{2}$ units are apically connected via O-bridges (O1 and $\mathrm{O} 5$ respectively), the $\mathrm{Cu}$-Dy closest intermetallic distance (3.42 ̊) falls midway in view of the ones between the smaller $\mathrm{Cu}(\mathrm{II})$ duo (3.22 $\AA$ ) and the larger Dy(III) pair (3.87 $\AA$ ), which is quite in accord with the structural predictions regarding heterometallic cubane species.

Similar PXRD profiles being recorded for two of the analogues unequivocally indicate the isostructural attributes for both the phases $\mathbf{1 D}$ and $\mathbf{2 L}$, in comparison to that of $\mathbf{1 L}$ (Fig. 3a), while similar IR stretching frequencies and elemental analyses for these merely reinstate the same fact.

Solid state CD spectra for two isostructural complexes (1D and $1 \mathrm{~L}$ ) arising out of different enantiomeric ligands (D and $\mathrm{L}$ respectively) are the reverse of each other as predicted (Fig. 3b). Although few in number, some chiral coordination complexes with interesting magnetic properties have been reported in the literature $;^{76-82}$ however, biomolecule-derived

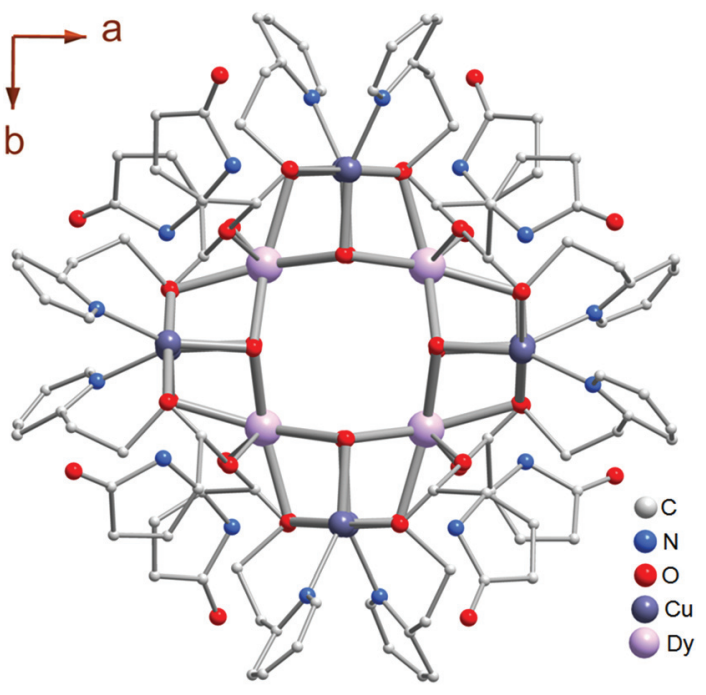

Fig. 1 Molecular structure of complex 1L (along crystallographic $c$-axis), as obtained from SC-XRD analyses presenting a highly symmetric Dy(III)-vertex sharing tetracubane-assemblage based dodecahedral $\mathrm{Dy}_{4} \mathrm{Cu}_{8}$ heterometallic cluster core.
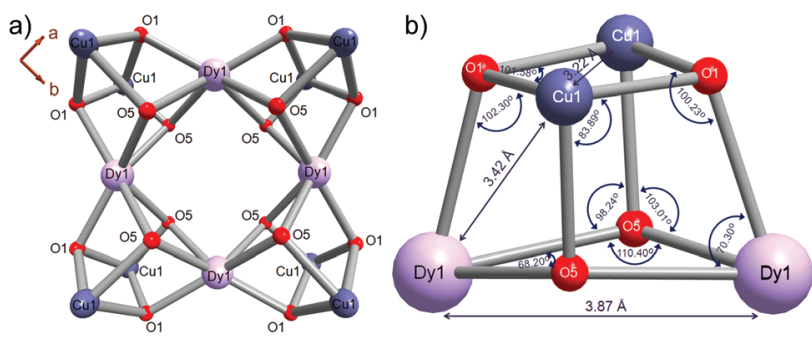

Fig. 2 (a) The Dy(III) vertex-sharing arrangement of four $\mathrm{Dy}_{2} \mathrm{Cu}_{2}$ cubanes to constitute a $\mathrm{Dy}_{4} \mathrm{Cu}_{8}$ dodecahedron architecture, viewed along c-axis; (b) intermetallic distances and bond angles spanned at different vertices of each of the four $\mathrm{Dy}_{2} \mathrm{Cu}_{2}$ constituent cubanes of the symmetric heterometallic core.

chirality based coordination complexes acting as molecular magnets have not yet been reported.

The direct-current (dc) magnetic susceptibilities of $\mathbf{1 L}$ and 2L have been measured in an applied dc magnetic field of 1000 Oe between 300 and $2 \mathrm{~K}$. The plots of $\chi_{\mathrm{M}}$ T versus $T$, where $\chi_{\mathrm{M}}$ is the molar magnetic susceptibility, are shown in Fig. 4a and $4 \mathrm{~b}$. At room temperature, the corresponding $\chi_{\mathrm{M}} T$ values equal to 3.19 and $59.87 \mathrm{~cm}^{3} \mathrm{~K} \mathrm{~mol}^{-1}$ for $2 \mathrm{~L}$ and $\mathbf{1 L}$ respectively (Fig. $4 \mathrm{a}$ and $4 \mathrm{~b}$ ) are in agreement with the expected values for eight uncoupled $\mathrm{Cu}^{\mathrm{II}}$ ions $\left(S=1 / 2, g=2, C=0.375 \mathrm{~cm}^{3} \mathrm{~K}\right.$ $\mathrm{mol}^{-1}$ ) for $\mathbf{2} \mathbf{L}$ and eight uncoupled $\mathrm{Cu}^{\mathrm{II}}$ ions altogether with four uncoupled Dy ${ }^{\mathrm{III}}$ ions $\left(S=5 / 2, L=5,{ }^{6} \mathrm{H}_{15 / 2}, g=4 / 3, C=\right.$ $14.18 \mathrm{~cm}^{3} \mathrm{~K} \mathrm{~mol}^{-1}$ ) for $1 \mathrm{~L}$ in the free-ion approximation.

For $2 \mathbf{L}$, the $\chi_{\mathrm{M}} T$ value remains unchanged with decreasing temperature until about $100 \mathrm{~K}$, where it sharply decreases to $0.21 \mathrm{~cm}^{3} \mathrm{~K} \mathrm{~mol}^{-1}$ at $2 \mathrm{~K}$. Considering the diamagnetic $\mathrm{Y}^{\mathrm{III}}$ ion, this decrease means the occurrence of antiferromagnetic inter- 

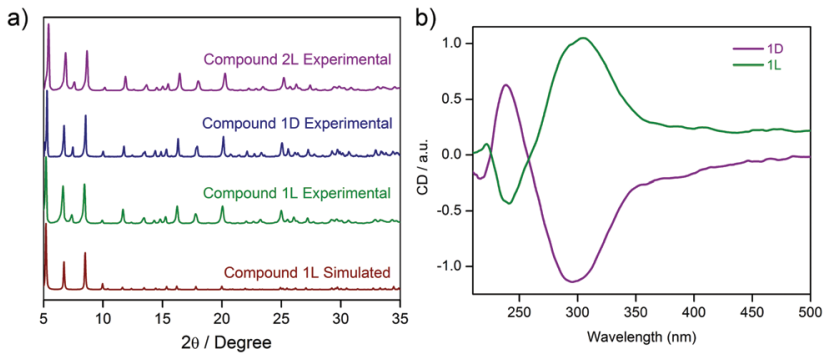

Fig. 3 (a) PXRD profiles suggesting a similar nature and phase purity for the three analogous $\mathrm{M}_{4} \mathrm{Cu}_{8}$ compounds reported herein, viz. 1L, 1D, and $2 \mathrm{~L}$ respectively, as compared to the simulated pattern for the L-PGA based cluster 1L; (b) solid-state CD spectra of $1 \mathrm{~L}$ and 1D; the two chiral clusters synthesized from PGA ligands with different chirality signatures, viz. L-PGA and D-PGA respectively.
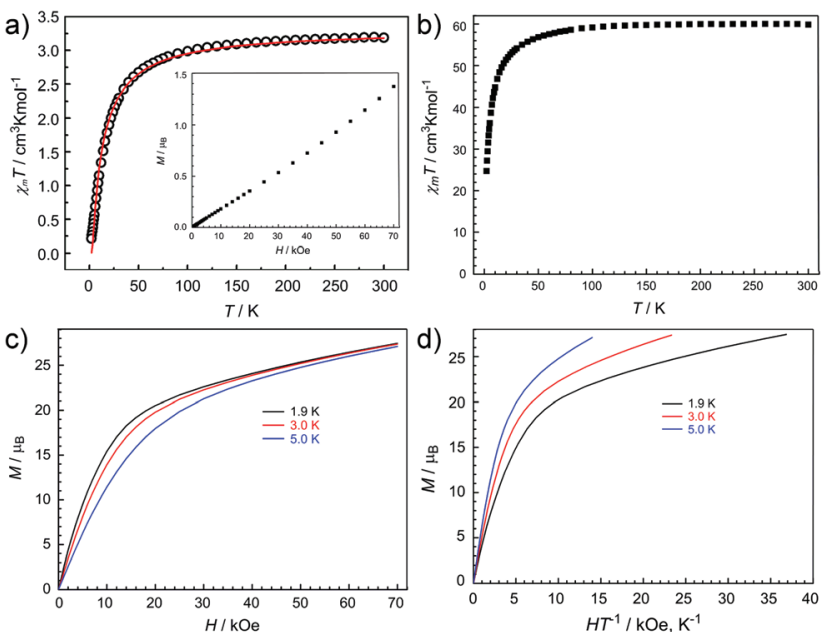

Fig. 4 Temperature dependence of $\chi_{\mathrm{M}} T$ in $2 \mathrm{~L}$ (left; a) and 1L (right; b) under 1000 Oe field, $\chi_{M}$ being the molar magnetic susceptibility. The red solid line corresponds to the calculated behavior of compound $2 \mathrm{~L}$. Inset of a: plot of field dependence of the magnetization (M) of $2 \mathrm{~L}$ at $2 \mathrm{~K}$; (c) Plots of $M$ vs. $H$ (left) and (d) the reduced magnetization $M$ versus $H / T$ (right) at the indicated temperatures for compound $1 \mathrm{~L}$.

actions among $\mathrm{Cu}^{\mathrm{II}}$ ions. In order to quantify the magnetic coupling, we simulated the magnetic data by using one $J$ coupling parameter. The experimental susceptibility data for $\mathbf{2 L}$ was fitted by $P H I$ program $^{83}$ using the isotropic one- $J$ model and the Hamiltonian $\hat{H}=-2 J \hat{S}_{1} \hat{S}_{2}$, where $J$ represents the exchange parameter between $\mathrm{Cu}^{\mathrm{II}}$ ions. The fit provides a set of parameters, $J=-5.71 \mathrm{~cm}^{-1}, g=2.10$ and the intermolecular antiferromagnetic interactions $z j=-0.5 \mathrm{~cm}^{-1}$. The variablefield magnetization measurement of $2 \mathrm{~L}$ at $1.8 \mathrm{~K}$ is shown in the inset of Fig. 4a. Moreover, the field-dependent magnetization at low temperatures reveals a steady increase approaching the value of $1.4 \mu_{\mathrm{B}}$ for $2 \mathrm{~L}$ at $70 \mathrm{kOe}$ without saturation (Fig. 4a, inset). The profile of the $M$ vs. $H$ plot confirms the existence of antiferromagnetic interactions within the clusters.

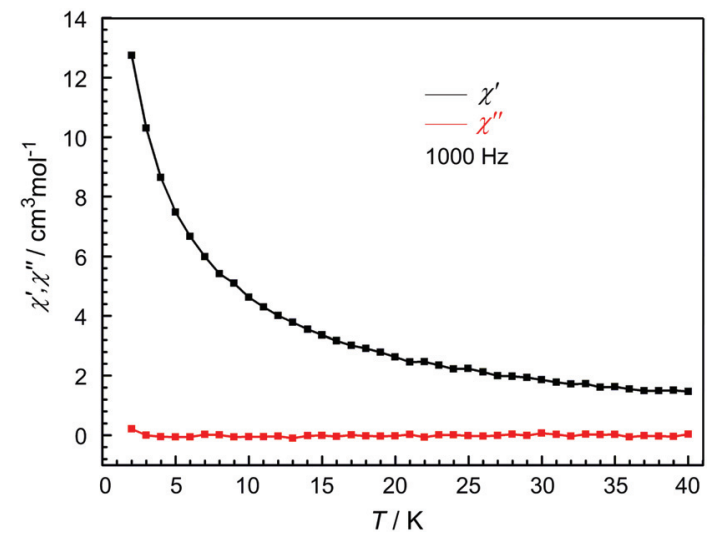

Fig. 5 Temperature dependence of in-phase $\left(\chi^{\prime}\right)$ (black) and out-ofphase $\left(\chi^{\prime \prime}\right)($ red) ac susceptibilities of $1 \mathrm{~L}$ at $1000 \mathrm{~Hz}$ in zero dc field and 3 Oe ac field.

Upon cooling, the $\chi_{\mathrm{M}} T$ value for $1 \mathrm{~L}$ gradually decreases from 300 to $50 \mathrm{~K}$, subsequently followed by further rapid decline to reach the minimum of $24.70 \mathrm{~cm}^{3} \mathrm{~K} \mathrm{~mol}^{-1}$ at $2 \mathrm{~K}$. This thermal evolution may be ascribed to the depopulation of the Stark sublevels and/or significant magnetic anisotropy present in Dy-containing systems, ${ }^{84,85}$ and does not preclude very weak antiferromagnetic magnetic interactions between spin carriers.

Magnetization $(M)$ data for $\mathbf{1 L}$ were collected in the 0-70 kOe field range below $5 \mathrm{~K}$. The field dependence of the magnetization $(M)$ shows that $\mathrm{M}$ increases smoothly with increasing applied dc field without saturation even at $7 \mathrm{~T}$ (Fig. 4c), which is ascribed to the anisotropy and the crystalfield effect. ${ }^{86}$ Furthermore, The non-superimpose $M$ vs. $H / T$ plot (Fig. 4d) also indicates the presence of significant magnetic anisotropy and/or low-lying excited states.

In order to investigate the dynamics of the magnetization, temperature dependence of the in-phase $\left(\chi^{\prime}\right)$ and out-of-phase $\left(\chi^{\prime \prime}\right)$ alternating current (ac) susceptibility measurements were carried out at $1000 \mathrm{~Hz}$ in zero dc field and 3 Oe ac field for $\mathbf{1 L}$. However, the absence of signals in the out-of-phase magnetic susceptibility operating in a 3.0 Oe ac field and a zero dc field (Fig. 5) indicates the lack of slow magnetic relaxation. This may be ascribed to the fast quantum tunneling phenomena.

\section{Conclusions}

In conclusion, magnetic studies on a biomolecule based symmetric chiral $\mathrm{M}_{4} \mathrm{Cu}_{8}$ cluster core are reported, which might be further exploited in the future to develop biocompatible magnetic materials from simple inexpensive precursors by coordination chemistry-driven self-assembly guided design principles. Considering the much less-explored domain of biomolecule-based magnetic materials, this report should help to make new inroads for the development of this new class of materials featuring the unique fusion of magnetism and biocompatibility. 


\section{Acknowledgements}

B. J. and S. M. are thankful to CSIR and IISER Pune for senior research fellowships respectively. We are grateful to IISER Pune for research facilities. DST (project no. GAP/DST/CHE-120083) is acknowledged for the financial support. DST-FIST (SR/ FST/CSII-023/2012) is acknowledged for micro-focus SC-XRD facility.

\section{Notes and references}

1 R. Mukherjee, Coord. Chem. Rev., 2000, 203, 151-218.

2 C. B. Aakeroy, N. Schultheiss, J. Desper and C. Moore, CrystEngComm, 2007, 9, 421-426.

3 A. Rajput and R. Mukherjee, Coord. Chem. Rev., 2013, 257, 350-368.

4 S.-i. Noro, K. Fukuhara, K. Sugimoto, Y. Hijikata, K. Kubo and T. Nakamura, Dalton Trans., 2013, 42, 11100-11110.

5 X.-D. Chen and T. C. W. Mak, Chem. Commun., 2005, 35293531, DOI: 10.1039/B505919D.

6 Z. Ma and B. Moulton, Coord. Chem. Rev., 2011, 255, 16231641.

7 P. J. Stang and B. Olenyuk, Acc. Chem. Res., 1997, 30, 502518.

8 C.-Y. Su, M. D. Smith and H.-C. zur Loye, Angew. Chem., Int. Ed., 2003, 42, 4085-4089.

9 K. Bhar, S. Das, S. Satapathi, P. Mitra, J. Ribas and B. K. Ghosh, Polyhedron, 2010, 29, 2041-2047.

10 M. Albrecht and R. Fröhlich, Bull. Chem. Soc. Jpn., 2007, 80, 797-808.

11 J. Krzystek, J. Telser, M. J. Knapp, D. N. Hendrickson, G. Aromí, G. Christou, A. Angerhofer and L. C. Brunel, Appl. Magn. Reson., 2001, 21, 571-585.

12 B. Bocquet, G. Bernardinelli, N. Ouali, S. Floquet, F. Renaud, G. Hopfgartner and C. Piguet, Chem. Commun., 2002, 930-931, DOI: 10.1039/B201859D.

13 Y. Zhang, Q. Wang, Y.-J. Xiao, J. Han and X.-L. Zhao, Polyhedron, 2012, 33, 127-136.

14 Y.-L. Miao, J.-L. Liu, J.-Y. Li, J.-D. Leng, Y.-C. Ou and M.-L. Tong, Dalton Trans., 2011, 40, 10229-10236.

15 S. Sakaue, A. Fuyuhiro, T. Fukuda and N. Ishikawa, Chem. Commun., 2012, 48, 5337-5339.

16 X.-L. Mei, R.-N. Liu, C. Wang, P.-P. Yang, L.-C. Li and D.-Z. Liao, Dalton Trans., 2012, 41, 2904-2909.

17 M. Du, C.-P. Li, C.-S. Liu and S.-M. Fang, Coord. Chem. Rev., 2013, 257, 1282-1305.

18 B. Joarder, S. Mukherjee, S. Xue, J. Tang and S. K. Ghosh, Inorg. Chem., 2014, 53, 7554-7560.

19 M. Du, X.-J. Jiang and X.-J. Zhao, Inorg. Chem., 2006, 45, 3998-4006.

20 Y.-W. Li, D.-C. Li, J. Xu, H.-G. Hao, S.-N. Wang, J.-M. Dou, T.-L. $\mathrm{Hu}$ and X.-H. Bu, Dalton Trans., 2014, 43, 1570815712.

21 D.-M. Chen, N. Xu, X.-H. Qiu and P. Cheng, Cryst. Growth Des., 2015, 15, 961-965.
22 A. A. Schilt, J. Am. Chem. Soc., 1960, 82, 3000-3005.

23 C. Palomo, M. Oiarbide and J. M. García, Chem. - Eur. J., 2002, 8, 36-44.

24 I. J. Hewitt, J. Tang, N. T. Madhu, C. E. Anson, Y. Lan, J. Luzon, M. Etienne, R. Sessoli and A. K. Powell, Angew. Chem., Int. Ed., 2010, 122, 6496-6500.

25 S. K. Langley, B. Moubaraki and K. S. Murray, Inorg. Chem., 2012, 51, 3947-3949.

26 A. Caneschi, D. Gatteschi, R. Sessoli, A. L. Barra, L. C. Brunel and M. Guillot, J. Am. Chem. Soc., 1991, 113, 5873-5874.

27 R. Sessoli, H. L. Tsai, A. R. Schake, S. Wang, J. B. Vincent, K. Folting, D. Gatteschi, G. Christou and D. N. Hendrickson, J. Am. Chem. Soc., 1993, 115, 18041816.

28 R. Sessoli, D. Gatteschi, A. Caneschi and M. A. Novak, Nature, 1993, 365, 141-143.

29 D. Gatteschi, A. Caneschi, L. Pardi and R. Sessoli, Science, 1994, 265, 1054-1058.

30 P. Zhang, L. Zhang and J. Tang, Dalton Trans., 2015, 44, 3923-3929.

31 L. Ungur, S.-Y. Lin, J. Tang and L. F. Chibotaru, Chem. Soc. Rev., 2014, 43, 6894-6905.

32 M. N. Leuenberger and D. Loss, Nature, 2001, 410, 789-793. 33 E. Coronado and P. Day, Chem. Rev., 2004, 104, 5419-5448.

34 A. Ardavan, O. Rival, J. J. L. Morton, S. J. Blundell, A. M. Tyryshkin, G. A. Timco and R. E. P. Winpenny, Phys. Rev. Lett., 2007, 98, 057201.

35 N. Roch, S. Florens, V. Bouchiat, W. Wernsdorfer and F. Balestro, Nature, 2008, 453, 633-637.

36 L. Bogani and W. Wernsdorfer, Nat. Mater., 2008, 7, 179186.

37 M. Mannini, F. Pineider, C. Danieli, F. Totti, L. Sorace, P. Sainctavit, M. A. Arrio, E. Otero, L. Joly, J. C. Cezar, A. Cornia and R. Sessoli, Nature, 2010, 468, 417-421.

38 M. Urdampilleta, N.-V. Nguyen, J.-P. Cleuziou, S. Klyatskaya, M. Ruben and W. Wernsdorfer, Int. J. Mol. Sci., 2011, 12, 6656-6667.

39 J. D. Rinehart, M. Fang, W. J. Evans and J. R. Long, Nat. Chem., 2011, 3, 538-542.

40 K. Katoh, H. Isshiki, T. Komeda and M. Yamashita, Chem. - Asian J., 2012, 7, 1154-1169.

41 I. Imaz, M. Rubio-Martinez, J. An, I. Sole-Font, N. L. Rosi and D. Maspoch, Chem. Commun., 2011, 47, 7287-7302.

42 J. An, S. J. Geib and N. L. Rosi, J. Am. Chem. Soc., 2009, 131, 8376-8377.

43 R. Schibli, R. La Bella, R. Alberto, E. Garcia-Garayoa, K. Ortner, U. Abram and P. A. Schubiger, Bioconjugate Chem., 2000, 11, 345-351.

44 F. Pu, X. Liu, B. Xu, J. Ren and X. Qu, Chem. - Eur. J., 2012, 18, 4322-4328.

45 N. Metzler-Nolte, Angew. Chem., Int. Ed., 2001, 40, 10401043.

46 Y. Wang, C. Zhang, H. Li, G. Zhu, S.-S. Bao, S. Wei, L.-M. Zheng, M. Ren and Z. Xu, J. Mater. Chem. B, 2015, 3, 296-305. 
47 R. Tashiro and H. Sugiyama, J. Am. Chem. Soc., 2005, 127, 2094-2097.

48 A. S. Stephen and J. L. Donald, Smart Mater. Struct., 2011, 20, 094018.

49 P. P. Freitas and H. A. Ferreira, in Handbook of Magnetism and Advanced Magnetic Materials, John Wiley \& Sons, Ltd, 2007, DOI: 10.1002/9780470022184.hmm428.

50 X. Liu, L. Li, J. Sun, Y. Yan, X. Shu, B. Liu, W. Sha, H. Feng, S. Sun and J. Zhu, Inorg. Chem., 2012, 51, 188-192.

51 Z. Liu, W. He and Z. Guo, Chem. Soc. Rev., 2013, 42, 15681600.

52 K. Tanaka and K. Fukase, Org. Biomol. Chem., 2008, 6, 815828.

53 R. Noguchi, A. Hara, A. Sugie and K. Nomiya, Inorg. Chem. Commun., 2006, 9, 355-359.

54 B. Joarder, A. K. Chaudhari, S. S. Nagarkar, B. Manna and S. K. Ghosh, Chem. - Eur. J., 2013, 19, 11178-11183.

55 R. Vaidhyanathan, C. A. Bridges, D. Bradshaw and M. J. Rosseinsky, Cryst. Growth Des., 2010, 10, 4348-4356.

56 P. Espeau, P. Negrier, H. Allouchi and R. Ceolin, Cryst. Growth Des., 2011, 11, 3418-3423.

57 R. Noguchi, A. Sugie, Y. Okamoto, A. Hara and K. Nomiya, Bull. Chem. Soc. Jpn., 2005, 78, 1953-1962.

58 G. Abraham and D. Podell, in The Biological Effects of Glutamic Acid and Its Derivatives, ed. V. A. Najjar, Springer, Netherlands, 1981, ch. 11, vol. 1, pp. 181-190.

59 D. Chelius, K. Jing, A. Lueras, D. S. Rehder, T. M. Dillon, A. Vizel, R. S. Rajan, T. Li, M. J. Treuheit and P. V. Bondarenko, Anal. Chem., 2006, 78, 23702376.

60 D. B. Liss, M. S. Paden, E. S. Schwarz and M. E. Mullins, Clin. Toxicol., 2013, 51, 817-827.

61 T. Goetze, C. Gansau, N. Buske, M. Roeder, P. Görnert and M. Bahr, J. Magn. Magn. Mater., 2002, 252, 399-402.

62 M. R. Loebinger, P. G. Kyrtatos, M. Turmaine, A. N. Price, Q. Pankhurst, M. F. Lythgoe and S. M. Janes, Cancer Res., 2009, 69, 8862-8867.

63 G. Novitchi, W. Wernsdorfer, L. F. Chibotaru, J.-P. Costes, C. E. Anson and A. K. Powell, Angew. Chem., Int. Ed., 2009, 48, 1614-1619.

64 A. K. Chaudhari, B. Joarder, E. Rivière, G. Rogez and S. K. Ghosh, Inorg. Chem., 2012, 51, 9159-9161.

65 K. Liu, W. Shi and P. Cheng, Coord. Chem. Rev., 2015, 289-290, 74-122.

66 X.-H. Miao, S.-D. Han, S.-J. Liu and X.-H. Bu, Chin. Chem. Lett., 2014, 25, 829-834.
67 Y.-Z. Ma, L.-M. Zhang, G. Peng, C.-J. Zhao, R.-T. Dong, C.-F. Yang and H. Deng, CrystEngComm, 2014, 16, 667-683.

68 J. Rinck, G. Novitchi, W. Van den Heuvel, L. Ungur, Y. Lan, W. Wernsdorfer, C. E. Anson, L. F. Chibotaru and A. K. Powell, Angew. Chem., Int. Ed., 2010, 49, 7583-7587.

69 O. Iasco, G. Novitchi, E. Jeanneau, W. Wernsdorfer and D. Luneau, Inorg. Chem., 2011, 50, 7373-7375.

70 SAINT Plus, (Version 7.03), Bruker AXS Inc., Madison, WI, 2004.

71 G. M. Sheldrick, SHELXTL, Reference manual: version 5.1, Bruker AXS, Madison, WI, 1997.

72 G. M. Sheldrick, Acta Crystallogr., Sect. A: Fundam. Crystallogr., 2008, 64, 112-122.

73 L. Farrugia, WINGX version 1.80.05, University of Glasgow, 2009.

74 A. L. Spek, PLATON, A multipurpose crystallographic tool, Utrecht University, Utrecht, The Netherlands, 2005.

75 O. Kahn, molecular magnetism.

76 N. Domingo, P. Gerbier, J. Gómez, D. Ruiz-Molina, D. B. Amabilino, J. Tejada and J. Veciana, Polyhedron, 2003, 22, 2355-2358.

77 M.-L. Sun, J. Zhang, Q.-P. Lin, P.-X. Yin and Y.-G. Yao, Inorg. Chem., 2010, 49, 9257-9264.

78 R. Inglis, F. White, S. Piligkos, W. Wernsdorfer, E. K. Brechin and G. S. Papaefstathiou, Chem. Commun., 2011, 47, 3090-3092.

79 G. Novitchi, G. Pilet, L. Ungur, V. V. Moshchalkov, W. Wernsdorfer, L. F. Chibotaru, D. Luneau and A. K. Powell, Chem. Sci., 2012, 3, 1169-1176.

80 X.-L. Li, C.-L. Chen, Y.-L. Gao, C.-M. Liu, X.-L. Feng, Y.-H. Gui and S.-M. Fang, Chem. - Eur. J., 2012, 18, 1463214637.

81 G. Novitchi, G. Pilet and D. Luneau, C. R. Chim., 2012, 15, 937-942.

82 K. Wang, S. Zeng, H. Wang, J. Dou and J. Jiang, Inorg. Chem. Front., 2014, 1, 167-171.

83 N. F. Chilton, R. P. Anderson, L. D. Turner, A. Soncini and K. S. Murray, J. Comput. Chem., 2013, 34, 1164-1175.

84 M. L. Kahn, J.-P. Sutter, S. Golhen, P. Guionneau, L. Ouahab, O. Kahn and D. Chasseau, J. Am. Chem. Soc., 2000, 122, 3413-3421.

85 M. L. Kahn, R. Ballou, P. Porcher, O. Kahn and J.-P. Sutter, Chem. - Eur. J., 2002, 8, 525-531.

86 J. Tang, I. Hewitt, N. T. Madhu, G. Chastanet, W. Wernsdorfer, C. E. Anson, C. Benelli, R. Sessoli and A. K. Powell, Angew. Chem., Int. Ed., 2006, 45, 1729-1733. 